Z. f. Elektroch. Bd. 14. 9. Oktober 1908. Nr. 4I. (S. 689-700.)

\title{
ÜBER DIE STICKOXYDBILDUNG IM HOCHSPANNUNGSBOGEN.
}

\author{
(II. Mitteilung.)
}

Von F. Haber und A. Koenig ${ }^{1}$ ).

(Aus dem Institut für Physikalische Chemie und Elektrochemie der Technischen Hochschule Karlsruhe.)

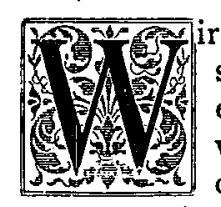
haben im Vorjabre unter demselben Titel in der Z. f. Elektroch. eine Abhandlung veröffentlicht, in welcher wir darzutun bemuht waren, daß die Stickoxydbildung im Hochspannungsbogen zwar so geführt werden $k a n n$, dab sie wesentlich ein rein thermisches $\mathrm{Phänomen}$ ist, daB aber gerade diese Ausführungsweise theoretisch nicht empfehlenswert ist. Unsere Arbeitsweise trat demgemå in Gegensatz zu der Arbeitsweise, die man fruher befolgt hat. Auf die kurzeste Form gebracht, kennzeichnet sich die Absicht älterer Versuche dahin, daß man die gasförmige Leiterstrecke möglichst boch zu erhitzen und dann möglichst rasch abzuschrecken bestrebt war, während wir darauf ausgingen, die gasförmige Leiterstrecke während des Stromdurchganges zu kühlen, so daß eine extreme, sehr hohe Temperatur derselben überhaupt nicht zustande kam. Bei extrem hoher Temperatur werden, wie wir uns zu zeigen bemüht haben, die primären elektrischen Wirkungen durch die übergelagerten thermischen Erscheinungen verdeckt. Bei unserer Arbeitsweise findet diese Erscheinung nicht statt. Alle unsere Versuche waren mit Stickstoff - Sauerstoffmischungen ausgeführt und lieferten zwar relativ sehr hohe, aber doch vom quantitativen Umsatz des Stickstoffes und Sauerstoffes zu Stickoxyd weit entfernte Ergebnisse. Wir äußerten die Vermutung, daß es sich hier um ein elektrisches Gleichgewicht handeln möchte, und wir haben diese Vermutung inzwischen durch neue Versuche bestätigt. Man erreicht, wie im folgenden gezeigt wird, dieselben Gehalte, ausgehend von Stickoxyd, die wir, ausgehend von Stickstoff-Sauerstoffmischungen, erhalten haben. Diese Gehalte erweisen sich von den elektrischen Versuchsbedingungen abhängig. Man gelangt also zu demselben stationären Zustand von beiden Seiten her. Wir bezeichnen diesen Zustand als elektrisches Gleichgewicht im Gegensatz zu dem thermischen Gleichgewichte. Während die Lage des thermischen Gleichgewichtes der drei Gase, Stickstoff, Sauerstoff und Stickoxyd, nur durch die Temperatur und die Massenwirkungen gekennzeichnet ist, verstehen wir unter dem elektrischen Gleichgewichte

I) Die erste Mitteilung findet sich in der $Z$. $f$. Elektroch. 13, 725 (1907). ein solches, bei welchem für den stationären Zustand noch ein bestimmter elektrischer Energieaufwand in der Zeiteinheit erforderlich ist, dessen Betrag für die Größe der Abweichung vom thermischen Gleichgewichtszustande bestimmend ist ${ }^{1}$ ). Bezeichnen wir mit $K_{t}$ die Gleichgewichtskonstante im gewöhnlichen Sinne, also

und kennzeichnen wir mit

$$
K_{t}=\frac{(N O)}{\left(N_{2}\right)^{1 / 2}\left(O_{2}\right)^{1 / 2}}
$$

$$
K_{e}=\frac{\left(N O^{\prime}\right)}{\left(N_{2}\right)^{1 / 2}\left(O_{2}{ }^{\prime}\right)^{1 / 2}}
$$

das Zusammensetzungsverhältnis beim Aufwande eines bestimmten, von den Versuchsbedingungen abhängigen Betrages elektrischer Energie in der Zeiteinheit, so ist unter der Voraussetzung der Temperaturgleichheit in beiden Făllen die Arbeitsgröße $R T \ln \frac{K_{e}}{K_{t}}$ ein $\mathrm{Maß}$ der elektrischen Gleichgewichtsverschiebung, bezogen auf das Mol Stickoxyd. Offenbar kann $K_{e}$ ebensowohl kleiner als größer wie $K_{t}$ sein, je nachdem der Ionenstof 3 mehr der Bildung oder der Zerlegung des Stickoxyds zugute kommt Würde das letztere der Fall sein, so müßte man streben, die elektrischen $\mathrm{Er}$ scheinungen durch die thermischen zu verdecken, wie man es früher getan hat. Liegt es hingegen umgekehrt - und dies ist der von uns vertretene Standpunkt -, so muf man die thermischen Erscheinungen zurückdrängen, um die elektrischen zur Geltung kommen zu lassen.

Das Verhaltnis des elektrischen Gleichgewichtszustandes zum thermischen hängt von der Geschwindigkeit $a b$, mit welcher (die Bildung und) der Zerfall des Stickoxyds bei der Versuchstemperatur auf nichtelektrischem Wege verläuft. Zwei Grenzfälle bieten sich zunächst der Betrachtung dar. Befinden wir uns in einem Temperaturgebiete, in welchem die (Bildungs- und) Zerfallsgeschwindigkeit des Stickoxyds praktisch Null ist, so wird die Lage des elektrischen Gleichgewichtes, welche durch die Konstante $K_{\varepsilon}$ gekennzeichnet ist, lediglich davon abhängen,

I) Die Abhängigkeit der Lage des elektrischen Gleichgewichtes von den einzeinen Formen der Gasentladung wird in dieser Mitteilung noch nicht bebandelt. 
in welchem Maße der Elektronenstoß bildend und zersetzend auf Stickoxyd einwirkt. Befinden wir uns anderseits in einem Temperaturgebiete, in welchem die Zerfallsgeschwindigkeit des Stickoxyds überaus großl ist, so wird das elektrische von dem thermischen Gleichgewichte, also die Konstante $K_{e}$ von der Konstante $K_{t}$ nicht merklich verschieden sein können, weil jeder elektrisch hervorgebrachte Mehrgehalt an Stickoxyd durch augenblicklichen Rückgang auf die Konzentration des thermischen Gleichgewichtes verschwindet. Daraus folgt, daß sich die thermischen Erscheinungen zugunsten der elektrischen nur zurückdrängen lassen, wenn man in einem Temperaturgebiete arbeitet, in welchem die Zerfallsgeschwindigkeit des Stickoxyds noch eine vergleichsweise geringe ist. Wir begründen im Fortgang dieser Mitteilung durch Versuche, daß unter unseren Arbeitsbedingungen das Stickoxyd in der Tat meBbar langsam in der Entladungsbahn zerfällt. Diese Darlegung bildet eine wesentliche Stütze unseres früheren, auf rechnerischem Wege gewonnenen Ergebnisses, daß in unserem gekühlten Hochspannungsbogen die Temperatur von $3000{ }^{\circ} \mathrm{C}$. nicht erreicht wird.

Je tiefer wir mit der Temperatur herabgehen, um so mehr sind wir gegen den thermischen Rückzerfall des Stickoxyds gesichert. Aber es scheint nach dem, was uber diese Verhältnisse in der physikalischen Literatur zu finden ist, daß der elektrische Arbeitsaufwand zur Hervorbringung der Gasionisation durch Stoß erheblich wăchst, wenn wir mit der Temperatur unter Weißglut heruntergehen. Es wird also ein günstigstes Gebiet der Temperatur geben, welches nach oben hin durch den Bereich rapiden thermischen Stickoxydzerfalles, nach unten hin durch das Gebiet hoher Ionisierungsarbeit begrenzt ist.

Um die Temperaturverhältnisse in unserem Entladungsrohr unmittelbar anschaulich zu machen, haben wir eine Reihe von Versuchen ausgeführt. Zunächst wurde ein Platindraht von 0,0 I mm Dicke in die Mitte des Rohres gebracht, welches bei diesem Versuche, wie überhaupt bei allen in dieser Abhandlung mitgeteilten Experimenten, genau der Fig. 4I 7 unserer ersten Mitteilung entsprach. Der feine Platindraht war so angeordnet, daß er gerade und horizontal durch die Mitte des Entladungsrohres lief, dessen Längsachse vertikal war. Dies wurde erreicht, indem ein offener Ring von etwas stärkerem Platindraht, auf welchen der dünne Draht diametral gespannt und gelötet war, in das Quarzrohr eingeschoben wurde, an dessen Wandung er sich mit leichter Federung glatt anlegte. Beim Durchgang der Entladung durch das Rohr gluhte dieser Draht schon bei kleinen Stromstärken hell, er schmolz aber erst, als die Stromstärke auf o,2 Amp. gesteigert wurde. Der Druck bei diesem Versuche betrug rund Ioo $\mathrm{mm}$ Quecksilber. Dickere Platindrähte schmolzen auch bei o,4 Amp. unter demselben Drucke nicht, weil sie offenbar durch Ableitung an die Quarzwand zu viel Wärme verloren. Gastemperatur und Platintemperatur sind auch bei Verwendung des Drahtes von o, or mm Stärke, bei welchem die Wärmeableitung durch die kälteren Drahtenden kaum mehr ins Gewicht fällt, nicht gleich zu setzen. Denn die Wärmekapazität des Platins ist im Vergleich zu der des Gases zu groß, als dab der feine Draht den thermischen Aenderungen des der Wechselstromwirkung unterworfenen Gases momentan folgte ${ }^{1}$ ). Aber die Gasbewegung, auf deren Heftigkeit wir in folgendem noch eingehen, ist so stark, daß der Unterschied nicht sehr groß werden kann, und es ist jedenfalls zu schließen, daß bei unseren Versuchen aber Stickoxydbildung und -Zersetzung; bei welchen die Stromstärke mit o,35 Amp. ihren Höchstwert erreichte, der Platinschmelzpunkt nicht um Tausende, sondern nur um Hunderte von Graden uberschritten wurde. Dasselbe Resultat läßt sich aus dem Ergebnis von Versuchen folgern, bei denen Auerstrumpfmasse unabgebrannt in das Entladungsrohr gebracht und dort der Wirkung der Entladungen bei Stromstärken bis 0,4 Amp. unter $100 \mathrm{~mm}$ Druck ausgesetzt wurde. Die veraschte Masse zeigte keinerlei Schmelzung, obwohl die höchst feinfaserige Struktur der Edelerdenasche eine sehr kleine Wärmeableitung verbürgt. Auch färbte sich die Entladungsstrecke nicht etwa mit den Dämpfen der Erden 2).

Im Hinblick auf die Rechnungen nach den Warburgschen Ansätzen, die wir in unserer ersten Mitteilung kurz skizziert haben, und deren genauere Durchführung in der Karlsruber

I) Der durch die stärkere Strahlung des Platins bedingte Unterschied seiner Temperatur gegen die Gastemperatur ist bei der Drahtdicke von o, OI $\mathrm{mm}$ sicherlich nicht groß. Denn man weiß aus Messungen von Flammentemperaturen mit Thermoelementen verschiedener Dicke, daß man mit so dünnen Drähten der Flanmentemperatur sehr nahe kommt.

2) Uebrigens sind Versuche mit Auermasse sehr geeignet, die äußerst heftige Gasbewegung zu zeigen, welche durch den Wechselstromdurchgang in dem Entladungsrohr eintritt. Denn einzelne lose Teilchen der Auermasse tanzen mit der größten Heftigkeit in dem Rohr, bis sie der Zufall früher oder später herausschleudert. Die Ursache dieser Bewegung, auf die wir die Vorstellung eines isothermen Gebietes in der Mitte unseres Rohres gegründet haben, liegt offenbar darin, daß die axialen Gasschichten in jeder Halbperiode durch Erwärmung beftig ausgedehnt werden, während die unmittelbar an der Quarzwand anliegenden Gasschichten nicht erheblich heiß werden. Die Reibung dieser sich ungleich rasch ausdehnenden Schichten muß die Bewegung notwendig zu einer wirbelnden machen. 
Dissertation von A. Koenig (1907) sich findet, wollen wir nicht unterlassen, dem Vorstehenden die Bemerkung hinzuzufügen, $\mathrm{da} B$ eine Verdoppelung der in unser Entladungsrohr hineingeschickten elektrischen Energie keineswegs einer Steigerung der Temperatur auf das Doppelte entspricht. Denn man sieht direkt, daß, wenn wir die Stromstärke und mit ihr die in das Entladungsrohr hineingesandte Energie vermehren, die leuchtende Entladung näher. an die kalte Quarzwand heranrückt, so daB die nicht leuchtende Zone, auf deren relativ schlechtem Wärmeleitungsvermögen das Heißwerden des Entladungskernes beruht, schmaler wird und größere Warmemassen dementsprechend durchzulassen vermag. Die Temperaturänderungen werden also kleiner sein, als die Aenderungen der hineingeschickten Energie. Im gleichen Sinne werden etwaige Wärme verbrauchende Vorgänge wirken, welche mit steigender Temperatur im Gase auftreten, welches die Entladungsbahn passiert. Diese Vorgänge verdienen noch einige Worte. Wir haben in unserer ersten Mitteilung den Nernstschen ${ }^{1}$ ) Ausdruck für das Gleichgewicht der Reaktion:

$$
\mathrm{N}_{2}+\mathrm{O}_{2} \mathrm{NO}
$$

bis zu Stickoxydkonzentrationen von $\mathbf{2 5} \%$ tabellarisch dargestellt, um den Leser instand zu setzen, die zu jedem Stickoxydgebalte bezw. zu jedem Werte der Gleichgewichtskonstante theoretisch zugehörige Temperatur mit einem Blicke zu erkennen. Wir haben uns keiner Täuschung darüber hingegeben, daß die abnorm hohen Temperaturen, welche sehr hohen Stickoxydgehalten entsprechen, keinerlei Sicherheit besitzen. Wir haben insbesondere auf die Möglichkeit hingewiesen, daß bei den extremen Temperaturen eine starke Volumenionisation der Gase möglich erscheint, und daß bei erheblicher Ionisationswärme eine wesentliche Verschiebung der Gleichgewichtslage dadurch bedingt sein kann. Auch andere qualitative Aenderungen erscheinen bei den extremen Temperaturen möglich. Nach einer Ueberschlagsrechnung von Nernst ${ }^{2}$ ) ist von $2000^{\circ} \mathrm{C}$. aufwärts ein merklicher mit der Temperatur

I) Wenn wir neben diesem zweigliedrigen Ausdruck den praktisch damit identischen zweigliedrigen Ausdruck benutzt haben, welchen $\mathrm{Haber}$ in seiner "Thermodynamik technischer Gasreaktionen " angibt, so geschah dies lediglich, weil der Nerustsche Ausdruck in der Wiedergabe in der Z. f. Elektroch. durch einen Druckfehler entstellt war. Das Verdienst, das thermische Stickoxydgleichgewicht erforscht und zuerst durch eiven zweigliedrigen Ausdruck in brauchbarer Forn als Temperaturfunktion dargestellt zu haben, gebïhrt ausschließlich Herrn Nernst (vergl. Göttinger Nachrichten 1904, 26r).

2) Z. f. Elektroch. 9, 891 u. 892 (1903). Vergl. Fischer und Braehmer, Berl. Ber. 39, I, 966 (xgo6). wachsender Ozongehalt des Sauerstoffes denkbar und auch Spaltung des Sauerstoffes in Atome, auf welche Herr Nernst gelegentlich bei mündlicher Unterhaltung hinwies, kommt bei den extremen Temperaturen in Betracht. Der monomolekulare Zerfall des Stickoxyds in Stickstoff - und Sauerstoffatome bei Lichtbogentemperatur, auf den Le Blanc und Nüranen ${ }^{1}$ ) aus ihren Versuchen schließen, kann als ein Hinweis auf die Atomspaltung der Elementargase gelten. Inzwischen ist im hiesigen Laboratorium durch Versuche von Haber und Hodsman ein Umstand ans Licht gekommen, welcher lehrt, daß qualitative Veränderungen des Sauerstoffes schon bei einer unerwartet niedrigen Temperatur merkbar werden. Bei diesen Versuchen hat sich nämlich gezeigt, daß die Dissoziation der Kohlensäure in der Kohlenoxydsauerstoffflamme nicht dann am höchsten gefunden wird, wenn man das stöchiometrische Gemisch der beiden Gase zur Flammenbildung benutzt, sondern wenn man einen Kohlenoxydüberschuß anwendet. $\mathrm{Ob}$ hier Ozonbildung, Atomspaltung oder elektrische Dissoziation des Sauerstoffes die Ursache der Erscheinung bildet, läßt sich noch nicht sagen, aber in jedem Falle muß man schließen, daß unter Wärmeabsorption Sauerstoff-Formen in der Gasmasse auftreten, welche wir nicht auf gewöhnliche Temperatur durch Abschreckung herabzubringen vermögen. Die Temperatur der Kohlenoxydknallgasflamme läßt sich danach schätzen, daf ein Iridiumblech von $0,02 \mathrm{~mm}$ Dicke und $2 \mathrm{~mm}$ Breite in ihr beginnende Schmelzung zeigt. Wir können also nur einige bundert Grad über dem Iridiumschmelzpunkt von $2300^{\circ} \mathrm{C}$. uns befinden: Auch läßt sich experimentell zeigen, $\mathrm{da} \beta$ in dieser Flamme die Stickoxydbildung aus den Elementen noch meßbar langsam erfolgt. Wir sind also jedenfalls noch unter $3000^{0} \mathrm{C}$. Diese Beobachtungen lassen die rechnerische Extrapolation des thermischen Gleichgewichts von $\mathrm{N}_{2}, \mathrm{O}_{2}$ und $\mathrm{NO}$ auf Temperaturen über 3000 ${ }^{0} \mathrm{C}$. bedenklich erscheinen, weil wir über die am Gleichgewicht teilnehmenden Sauerstoffformen und die mit ihrem Uebergang in molekularen Sauerstoff verknüpften Wärmen nicht Bescheid wissen. Wir glauben deshalb nicht, daß sich über das thermische Stickoxydgleichgewicht im ungekühlten Hochspannungsbogen, wie er, von unserer Arbeitsweise abgesehen, allgemein benutzt worden ist, eine glaubhafte Angabe machen läßt. Bei Luftflammen insbesondere sind die Stickoxydgehalte, die man gewinnt, wie wir schon früher betont haben, Ergebnis thermischer Nachwirkungen. Für unseren gekühlten Hochspannungsbogen aber hat die thermische Rechnung auf Grund der

I) Z. f. Elektroch. 13, 297 (1907). 
Nernstschen Bestimmungen die große Bedeutung, daß sie uns das Maß der elektrischen Gleichgewichtsverschiebung und damit den Vorteil erkennen labt, den wir durch die Zurückdrängung der thermischen Einflüsse hinter die elektrischen gewinnen. Folgende Ueberlegung wird dazu dienen, diesen Zusammenluang in das rechte Licht $z u$ setzen. Wie weiterhin experimentell gezeigt wird, läßt sich mit Sicherheit in unserem Entladungsrohr bei der Stromstärke von I 20 Milliampere, bei welcher ein Platindraht von o, or mm Dicke noch bei weitem nicht schmilzt, ein Stickoxydgehalt von $81 \%$ unter $100 \mathrm{~mm}$ Druck in halftigem Gemisch von Sauerstoff und Stickstoff erzeugen. Dies entspricht einer Konstante $K_{e}$ von o,186. Auf der anderen Seite entnimmt man der in unserer ersten Arbeit mitgeteilten Tabelle, daß dic Konstante des thermischen Gleichgewichtes $K_{t}$ erst für die den Platinschmelzpunlat erheblich übersteigende Temperatur von $193^{\circ} \mathrm{C}$. den Wert 0,025 erreicht, der 7,5 mal kleiner ist. Das Verhältnis $K_{e} / K_{t}$ ist also unter diesen Versuchsbedingungen größer als 7,5. Steigern wir die Stromstärke und damit die Temperatur, so wächst zwar der Stickoxydgehalt, der erreicht wird. Das Verbăltnis $K_{\bullet} / K_{t}$ aber nähert sich dem Werte I, den es notwendig erreicht, sobald wir in das Gebiet außBerst großer thermischer Zerfallsgeschwindigkeit des Stickoxyds gelangen. Wir finden mit den stärksten von uns benutzten Entladungen $\mathrm{I}_{4} 1 / 2 \% N O$ bei hälftigem Gemisch der Elementargase, entsprechend einem Werte $K_{e}=0,34$. Nehmen wir für die Temperatur der Entladungsbahn den Höchstwert niedrig, nämlich mit $2600{ }^{\circ} \mathrm{C}$. an, so beträgt die Konstante des thermischen Gleich. gewichtes $K_{t}=0,077$, und das Verhältnis $K_{e} / K_{t}$ ist nur noch 4,4 . Der wirkliche Wert dürfte noch kleiner sein. Der vorstehend erklärte Zusammenhang zwischen Stromstärke und Stickoxydkonzentration tritt in unserer früheren Mitteilung nicht in allen Fällen so deutlich in die Erscheinung. Der Grund ist darin zu sehen, daß sich bei unserer früheren Versuchsweise bei den Drucken in der Nähe von Ioo mm die Bogenspannung mit fallender Stronstärke oft sehr stark erhohte, wie aus unseren Versuchszahlen zu entnehmen ist. Die geschicktere Anordnung, die wir im Fortgang der hier beschriebenen Versuche gewählt haben, bestand darin, daß wir an Stelle des Ohmschen Widerstandes im Primärkreise des Wechselstromtransformators eine große Drosselspule mit regulierbarem Luftspalt verwendeten. Dadurch wurde nicht nur die Benutzung jedes Ballastwiderstandes im sekundären Stromkreis entbehrlich, sondern es wurde auch ein viel ruhigeres Brennen des Bogens bei relativ niedrigerer Spannung erreicht.
Nachdem wir früher Nernststifte als Elektrodenenden besonders zweckmäBig gefunden hatten, aber Schwierigkeiten bei deren Befestigung an den von uns beschriebenen, teilweise gekühlten eisernen Zuführungen begegnet waren, die sich in Spannungsschwankungen aussprachen, gelang es jetzt mit Nernststiften als Elektrodenenden durch eine andere Befestigung derselben ruhige und mit gleich/örmiger Spannung brennende Bogen zuerhalten. Während wir früher auf die eiserne Stromzuführung einen Platindraht als Verlängerung auflöteten, auf diesen den hohlen Nernststift auffädelten und ilnn mit Kaolin an der Basis festkitteten - ein Verfahren, das häufig ein Abbröckeln und Springen der Nernststifte infolge der thermischen Wirkungen zur Folge hatte -, bohrten wir jetzt den Kopf der eisernen Zuführung zu einem Robrchen aus und kitteten nunmehr einen Nernststift mit Glas in diese Ausbohrung. Es wurden Nernststifte aus etwas weicherer Masse als früher gewăhlt.

Das Stickoxyd wurde aus Kupfer und mäBig konzentrierter Salpetersäure dargestellt und über destilliertem Wasser aufgefangen. Das Gas enthielt neben rund $92 \%$ Stickoxyd Stickstoff und etwas Stickoxydul. Es konnte also mit genügender Annäherung als hervorgegangen aus der stöchiometrischen Vereinigung gleicher Volumina Sauerstoff und Stickstoff betrachtet werden, so daß die Stickoxydkonzentration, welche beim Durchleiten desselben durch unser Versuchsrohr übrig blieb, unmittelbar mit derjenigen vergleichbar war, die beim Durchleiten eines Gemisches gleicher Raumteile Stickstoff und Sauerstoff entstand. Ein solches Gemisch annähernd gleicher Raumteile der beiden Elementargase haben wir bei dieser Gelegenheit nochmals zu Vergleichsversuchen verwendet. Auch wurden die fruheren Versuche mit Luft wiederholt. Die Ausführungsweise der Versuche war dieselbe, die wir in unserer ersten Mitteilung beschrieben haben ${ }^{1}$ ). Die Dauer jedes Versuches wurde so bemessen, daß durch die Pipette mehr als das Zehnfache ihres Gasinhaltes strömen mußte; die Zusammensetzung der Gasprobe entsprach so

I) Hinsichtlich der analytischen Methodik ist eine kleine Undeutlichkeit zu verbessern. Wir habeu bei der Beschreibung unserer analytischen Blindversuche (S. 733, reclite Spalte) von der Ammoniakmenge gesprochen, die ,, aus dem gleichen Quantum der zuvor verwendeten Kalilauge für sich entstand". Hier ist nicht gemeint, daß die Kalilauge anmoniakhaltig war, sondern es handelt sich um die Einwirkung des gleichen Quantums Kalilauge auf das gleiche Quantum Devardascher Legierung. Dabei ist letztere (Nitridgehalt) und nicht die Kalilauge die Quelle der im Blindversuche auftretenden geringen Ammoniakmenge, wie wir jetzt noch eigens dadurch festgestellt haben, daß wir die frühere $Z$ ahl mit derselben Legierung und verschiedenen Kalilaugen wiederfanden, ohne die Legierung aber mit Kalilauge allein kein $\mathrm{NH}_{3}$ gewannen. 
jedenfalls recht genau der des aus dem Lichtbogen austretenden Gases (abgesehen natürlich von der inzwischen erfolgten Oxydation des Stickoxyds zu Stickstoffdioxyd).

Wir geben zunächst sieben Versuclse an, bei denen die Reyulierung des Stromes nicht durch die Drosselspule, sondern noch, wie früher, durch einen Kurbelrheostat im Primärkreise geschah. Diese Versuche wurden alle mit Stickoxyd ausgeführt. Sie sind in der Tabelle I vereinigt.

Tabelle I.

Versuche mit Stickoxyd.

\begin{tabular}{|c|c|c|c|c|c|c|c|}
\hline 兽 & 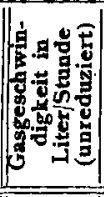 & 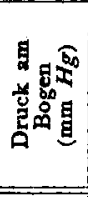 & 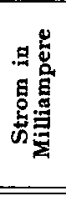 & 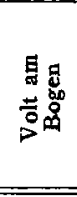 & 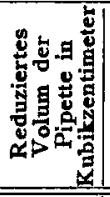 & 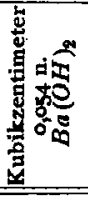 & 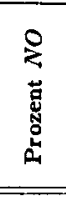 \\
\hline $\begin{array}{l}1 \\
2 \\
3 \\
4 \\
5 \\
6 \\
7\end{array}$ & $\begin{array}{l}0,7 \\
0,7 \\
0,7 \\
0,7 \\
0,7 \\
0,7 \\
0,7\end{array}$ & $\begin{array}{l}87 \\
87 \\
87 \\
85 \\
87 \\
85 \\
85\end{array}$ & $\begin{array}{l}200 \\
180 \\
210 \\
220 \\
370 \\
340 \\
355\end{array}$ & $\begin{array}{l}6600 \\
6700 \\
5700 \\
5700 \\
3900 \\
4000 \\
2400\end{array}$ & $\begin{array}{l}55,0 \\
50,7 \\
45,5 \\
53,8 \\
47,6 \\
56,4 \\
57,2\end{array}$ & $\begin{array}{l}6,89 \\
5,98 \\
5,63 \\
6,61 \\
570 \\
6,92 \\
6,82\end{array}$ & $\begin{array}{l}15,2 \\
14,3 \\
15,0 \\
14,9 \\
14.5 \\
14,9 \\
r_{4,5}\end{array}$ \\
\hline
\end{tabular}

Die Zahlen fur die Stickoxydkonzentration liegen denen, die wir früher, ausgehend von annähernd halftigem Gemisch erhalten und mitgeteilt haben, sehr nahe. Der wesentliche Vorteil, den der Ersatz der Widerstănde durch die Drosselspule brachte, spricht sich, wie man beim Vergleich der mit der Drosselspule ausgeführten Versuche der Tabelle 2 erkennt, nicht in den Konzentrationen, wohl aber sehr deutlich in den am Bogen herrschenden Spannungen aus.

Tabelle 2.

\begin{tabular}{|c|c|c|c|c|c|}
\hline 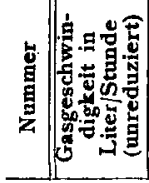 & 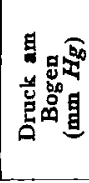 & 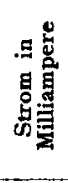 & 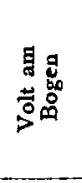 & 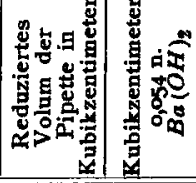 & 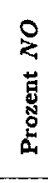 \\
\hline
\end{tabular}

\begin{tabular}{|c|c|c|c|c|c|c|c|}
\hline & & & Versuc & mit & uft. & & \\
\hline 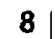 & 0,7 & 105 & 330 & 1800 & 71,9 & 6,24 & 10,5 \\
\hline & 0,7 & III & 320 & 1900 & 74 & 5,8 & 9,4 \\
\hline & 4,6 & 105 & $33^{\circ}$ & $I 800$ & $67, \mathbf{I}$ & $5,3 \mathrm{I}$ & \\
\hline II & 4,6 & 105 & $34^{\circ}$ & 1700 & 57,8 & 4,65 & 9,8 \\
\hline 12 & 4,6 & 105 & 340 & 1700 & 55,2 & 4,39 & 9,7 \\
\hline & 4,6 & 105 & 340 & 1700 & 69 & $5,4^{8}$ & \\
\hline 14 & 4,6 & 103 & 330 & 1700 & 67,2 & $5 \cdot 3^{\mathrm{I}}$ & 9,6 \\
\hline & 4,6 & 103 & $33^{\circ}$ & 1700 & 68,9 & 5.5 & 9,7 \\
\hline An & 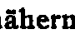 & nalt & 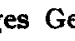 & tasca & $7.5 \%$ & 2, $5^{2}$ & $10 N_{2}$ \\
\hline I6 & 4,3 & IO4 & $34^{\circ}$ & I850 & 72,1 & $8,6 \mathrm{I}$ & 14,5 \\
\hline-6 & 4,3 & xo4 & $34^{\circ}$ & 1850 & 70,9 & 8,69 & $\mathbf{1} 4,9$ \\
\hline$\pi 8$ & 4,3 & IO2 & $34^{\circ}$ & $185^{\circ}$ & 68,8 & 8,4 & 14,8 \\
\hline IS & 4,3 & 102 & 335 & 1850 & 66,2 & 7,91 & 14,5 \\
\hline & 4,3 & & & 1850 & 54,7 & 6,6 & 14,6 \\
\hline & & & suche & nit & & & \\
\hline & 4, & $10_{4}$ & 34 & 1800 & 71,7 & 8,7 & $I_{4,7}$ \\
\hline & 4,3 & 103 & $\begin{array}{r}340 \\
240\end{array}$ & $\begin{array}{l}1800 \\
1760\end{array}$ & $\begin{array}{l}69,5 \\
68.2\end{array}$ & $\begin{array}{l}8,49 \\
8,21\end{array}$ & $\begin{array}{l}14,8 \\
146\end{array}$ \\
\hline & 4,3 & & & & & & \\
\hline
\end{tabular}

Die Versuche der Tabelle 2 sind übereinstimmend mit relativ großer Stromstarke ausgeführt. Die Versuche 8 bis is bestätigen die in unserer älteren Arbeit mitgeteilten (Tabelle 4 derselben), die unter gleichen Bedingungen ausgeführt sind. Ebenso bestätigen die Versuche I 6 bis 20 die in Tabelle 7 der alteren Arbeit angegebenen Zahlen. Schließlich lehren die Versuche $2 \mathrm{I}$ bis 23 in Verbindung mit den Versuchen I bis 7 , daß sich dieselben Werte von der Gegenseite einstellen.

Versuche mit niederer Stromstärke, die von beiden Seiten ausgeführt, zu demselben Gleichgewichte fahrten, sind in der Tabelle 3 vereinigt. Der Vergleich der Zahlen der Tabellen 2 und 3 macht den frōher besprochenen Einflub der Stromdichte bezw. der elektrischen Energie auf das Gleichgewicht mit einem Blicke deutlich. Bei den Versuchen der Tabelle I, bei denen außerordentlich hohe Spannungen zur Wirkung kamen, blieb offenbar auch bei kleinem Strom die hineingeschickte Energie zu grob, als daß der Prozentgehalt an Stickoxyd unter den Maximalwert von $141 \frac{1}{2} \%$ gesunken wäre.

Tabelle 3 .

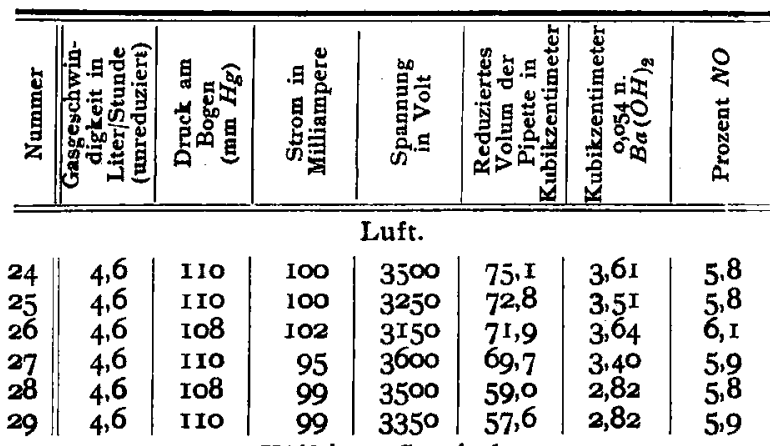

Hälftiges Gemisch.

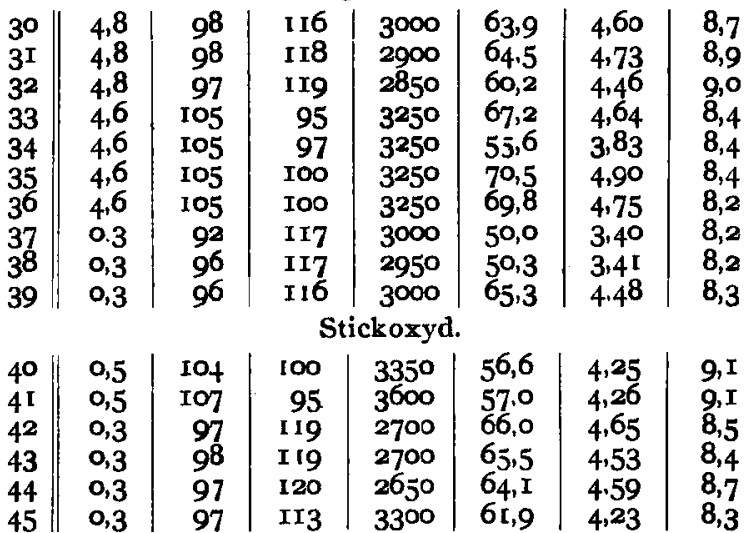

Die Tabelle 4 vereinigt Versuche mit rund I 20, 200 und 350 Milliampere, bei welchen die Durchtrittsgeschwindigkeit des in allen Fallen benutzten Stickoxyds durch das Entladungsrohr größer gewăhlt war. Um das der Stromstarke von 200 Milliampere entsprechende elektrische Gleichgewicht festzustellen, sind vier Versuche 
mit dieser Stromstärke bei sehr kleiner Strömungsgeschwindigkeit ausgeführt worden. Ihr Ergebnis stimmt, wie man leicht sieht, mit dem der älteren Versuche unserer ersten Mitteilung (Tabelle 7 derselben), die unter vergleichbaren Bedingungen mit hälftigem Stickstoff-Sauerstoffgemisch ausgeführt sind, mit genügender Annäherung uberein. Vergleicht man die in der Tabelle 4 mitgeteilten Zahlen mit denen der Tabellen 3 und 2, so sieht man, da 3 der Stickoxydgehalt, welcher über den Gleichgewichtswert hinaus unzersetzt bleibt, bei diesen Versuchen folgende Beträge hat:

$$
\begin{aligned}
& \text { Bei } 120 \text { Milliampere } 9 \% \text {, }
\end{aligned}
$$

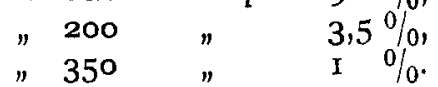

Tabelle 4 .

\begin{tabular}{|c|c|c|c|c|c|c|c|}
\hline $\begin{array}{l}\overline{\mathrm{U}} \\
\mathrm{g} \\
\mathrm{g}\end{array}$ & 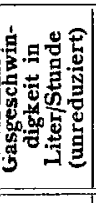 & 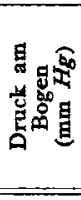 & 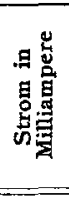 & 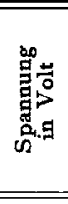 & 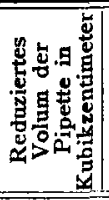 & 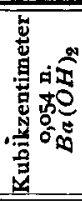 & 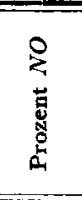 \\
\hline 46 & 5,0 & 100 & II9 & $245^{\circ}$ & 53,5 & 7,63 & 17,3 \\
\hline 47 & 5,0 & 99 & 120 & 2400 & 50,7 & 7,40 & 17.7 \\
\hline 48 & 5,0 & 98 & I25 & $245^{\circ}$ & 65,4 & 9,60 & 17,8 \\
\hline 49 & II & 107 & 200 & 2160 & 53,2 & 6,84 & 15,6 \\
\hline $5^{\circ}$ & I I & 105 & 200 & 2160 & 55,0 & 7,25 & 16,0 \\
\hline 51 & I I & 105 & 200 & 2160 & 68,0 & 14,29 & I5,7 \\
\hline $5^{2}$ & I I & 103 & 200 & 2160 & 64.9 & 13,67 & 15,75 \\
\hline 53 & 10,5 & III & 35 & 1900 & 72,3 & 9,10 & 15,2 \\
\hline 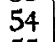 & 10,5 & 105 & 35 & 1850 & 67.7 & 8,60 & 15,4 \\
\hline J & IO, 5 & 106 & 35 & 1850 & 52,75 & 6,73 & 154 \\
\hline $5^{6}$ & 10,5 & IO9 & $35^{\circ}$ & I900 & 57,3 & $7,3^{8}$ & $\mathbf{I} 5,6$ \\
\hline 57 & 0,59 & 93 & 210 & 2160 & 政 & 9,12 & $\mathrm{II}, \mathrm{I}$ \\
\hline & 0,59 & & & & $6 r, 9$ & 9,69 & 11,7 \\
\hline 0 & $\begin{array}{l}0,59 \\
0.59\end{array}$ & $\begin{array}{r}\text { I0 } 5 \\
08\end{array}$ & 900 & $225^{\circ}$ & $\begin{array}{l}60,2 \\
50,5\end{array}$ & $\begin{array}{r}\mathrm{I}, 60 \\
8,26\end{array}$ & 12,0 \\
\hline & & & & & & & 12,2 \\
\hline
\end{tabular}

Hält man an der von uns bestrittenen Auffassungsweise fest, nach welcher die hohen, von beiden Seiten erreichten Stickoxydgehalte dem thermischen Gleichgewichte einer extrem hohen Temperatur entsprechen, so muß man eben dieser extremen Temperatur zuliebe annehmen, daß alles Stickoxyd, welches die Entladungsbahn passiert, momentan bis zur Gleichgewichtskonzentration zerfällt. Die Versuchsdaten der Tabelle 4 verlangen dann die Deutung, daB ein gewisser Bruchteil des Stickoxydes durch das Rohr schlüpft, ohne von der Wirkung der Entladung getroffen zu werden. Ein Durchschlüpfen des Gases wäre zunächst dann möglich, wenn die Gasgeschwindigkeit den Wert überschritte, bei welchem auf jede Rohrfälung noch mindestens eine volle Halbperiode des Wechselstromes entfallt. Von dieser Grenze bleiben jedoch die in der Tabelle 4 mitgeteilten Versuche auch bei ungünstigster Berechnungsweise noch weit $a b$. Bei einer Geschwindigkeit von I I Liter/Stunden (unreduziert) und bei roo Wechseln pro Sekunde, wie sie bei unseren Versuchen stattfanden, haben wir $0,03 \mathrm{ccm}$ (unreduziert)/Halbperioden. Das Volumen unseres Entladungsrohres zwischen der Gaszu- und -Abführung betrug, wie man aus den in unserer Abbildung angegebenen Maßen leicht ableitet, I, $17 \mathrm{ccm}$, also das 39 fache. Nehmen wir an, daß bei der Maximaltemperatur der einzelnen Halbperiode jedesmal die Gasmasse im Rohr auf den I5. Teil sinkt, so bleiben noch immer mindestens $21 / 2$ Halbperioden, welche der UeberschulB an Stickoxyd überdauert hat.

Eine andere Möglichkeit des Durchschlüpfens unzersetzten Stickoxyds bestände in einer Gleitung desselben längs der durch die Wirkung des umgebenden Wassers kalt gehaltenen Wandung. Aber diese Erklärung leuchtet besonders bei den Versuchen 46 bis 48 der Tabelle 4 sebr wenig ein. Denn bei diesen Versuchen müßten 10 $\%$ des ursprünglichen Gases unverändert längs der Wandung hindurchgeschlüpft sein, um das Ergebnis mit Hilfe dieser Vorstellung zu erklären. Nun muß man sich gegenwärtig halten, daß der Inhalt des Entladungsrohres einer doppelten Bewegung unterworfen ist. Einerseits befindet sich die ganze Gasmasse in vorwärtsströmender Bewegung, anderseits wird während jeder Halbperiode die Hauptmasse des Gases durch die große Temperaturänderung nach vorwärts und rückwärts aus dem Rohre ausgestoßen und danach wieder zurückgesogen. Berücksichtigen wir, was bei der früheren, absichtlich möglichst ungünstig geführten Rechnung nicht beachtet wurde, daB die Gase mit gewohnlicher Temperatur ein- und austreten, so entfallen bei der in den Versuchen 46 bis 48 innegehaltenen Gasgeschwindigkeit $14 \mathrm{cmm}$ auf eine Halbperiode des Stromes, während der Reaktionsraum, wie erwähnt, I, I $7 \mathrm{ccm}$, also das Achtzigfache beträgt. Es findet also $80 \mathrm{mal}$ ein Ausstoßen und Wiedereinsaugen der Hauptmasse des Rohrinhaltes statt, ehe derselbe vom Eintritt der Entladungsbahn endgültig zum Austritt derselben gelangt ist. Die Heftigkeit dieser Bewegung haben wir an einer fraheren Stelle dieser Notiz anmerkungsweise erläutert. Hierzu kommt, daß das Gas längs der Wände, wo es kalt bleibt, nach den Gesetzen der Strömung in Rohren sich viel langsamer bewegt, als der durch die Mitte - also im vorliegenden Falle durch die Entladungsbahn - strömende Anteil. In Erwägung dieser Verhältnisse wird man nicht annehmen dürfen, daß ein nennenswerter Prozentsatz der Gasmasse längs der Wände durch das Rohr zu schlapfen vermag, ohne in den Wirkungsbereich der Entladung zu gelangen. Dagegen lassen sich die Ergebnisse der in Tabelle 4 vereinigten Versuche durch die Annahme trägen thermischen Stickoxydzerfalles in der Entladungsbahn ungezwungen deuten. Dieser 
Schluß bestätigt die Vorstellung, welche wir aus dem Verhalten der Platindrähte in der Entladungsbahn über die dort herrschende Temperatur abgeleitet haben, und stützt die Vorstellung vom elektrischen Charakter des Gleichgewichtes, welches, wie wir im Eingang dieser Arbeit ausgeführt haben, nur im Gebiete trägen thermischen Stickoxydzerfalls vom thermischen verschieden gelegen sein kann.

Als mehr beiläufiges Ergebnis ist aus den Versuchen 46 bis $4^{8}$ herzuleiten, daß der Durchgang der Entladung durch das stickoxydreichere Gas relativ kleinere Spannungen erfordert.

Als wesentlichstes Ergebnis dieser Arbeit ist der Nachweis zu bezeichnen, daß wir die ungewöhnlich hohen Stickoxydgehalte von nahezu ro $\%$ bei Luft und $14 \frac{1}{2} \%$ bei halftigem Gemisch von beiden Seiten, und $z$ war bei Temperaturen der Entladungsbahn erhalten, die höchstens Hunderte, aber keinesfalls Tausende von Graden uber dem Platinschmelzpunkt liegen und einem Temperaturgebiet angehören, in welchem der Stickoxydzerfall noch relativ langsam geschieht. Der elektrische Charakter der von uns beobachteten Stickoxydgleichgewichte im gekühlten Hochspannungsbogen unter starkem Minderdruck dürfte damit bewiesen sein.

(Eingegangen: 27. August.)

\section{ÜBER DIE ZERSTÄUBUNG DER KATHODEN IN VERDÜNNTEN GASEN. Von B. Walter.}

F. Fischer und O. Hähnell) ziehen im Gegensatz zu V. Kohlschüter und seinen Mitarbeitern ${ }^{2}$ ) aus ihren Versuchen den Schluß, daß fär die Zerstäubung der Kathode einer Vakuumröhre das Gas in der letzteren, wenn es nicht beständige Verbindungen mit dem Elektrodenmaterial (z. B. Oxyde oder Nitride) bilde, vollkommen gleichgultig sei. Im besonderen haben sie die Zerstäubung in Wasserstoff und in Argon miteinander verglichen, indem zwei übereinstimmende, mit den beiden Gasen unter gleichem Druck gefüllte Röhren hintereinander in den. selben Stromkreis geschaltet wurden, und dabei für sämtliche elf von ihnen untersuchten Metalle fast genau die gleiche Zerstăubung gefunden. Die Beobachter glauben daher, die Auffassung Kohlschütters, daß dieser Vorgang auch bei den Edelgasen durch eine chemische Verbindung derselben mit dem Elektrodenmetall bedingt werde, nicht annehmen zu können.

Der letztere hat nun demgegenaber zwar schon betont, daß nach seiner Auffassung die zerstäubte Metallmenge nicht, wie die genannten Beobachter es getan haben, in Beziehung zu

I) Z. f. Elektroch. 14, 366 u. 433 (Igo8).

a) Z. f. Elektroch. 12, 365 u. 869 (1906); 14, 22 t u. 437 (Igo8). der durch die Röhre gehenden Elektrizitätsmenge, sondern zu der von der Kathode derselben verbrauchten elektrischen Energie, d. h. also zu dem Produkt aus Elektrizitätsmenge und Kathodenfall, zu setzen sei. Er hätte aber meines Erachtens noch weiter hinzufügen können, daß bei der Schaltung von Fischer und Hähnel zwar die Elektrizitătsmengen in den beiden hintereinander geschalteten Röhren die gleichen, der Kathodenfall aber in der Argonröhre stets kleiner ist als in der Wasserstoffröhre, wie ja auch die Beobachter selbst schon daraus geschlossen haben, daß die letztere Röhre beim Stromdurchgang stets wärmer wurde als die erstere. Bei gleicher verbrauchter elektrischer Energie ergibt sich demnach auch aus den Versuchen von $\mathrm{Fischer}$ und $\mathrm{Hăhnel}$ für Argon eine größere Zerstäubung als für Wasserstoff, wie die Kohlschüttersche Theorie es verlangt. Immerhin ist aber eine genauere Bestätigung der letzteren natürlich erst dann zu erwarten, wenn die Versuche mit einer Stromquelle durchgefahrt werden, bei der sich Strom und Spannungsabfall beide genau messen lassen, Versuche, die ja auch von dem Begründer der Theorie demnächst in Angriff genommen werden sollen.

Hamburg, September 1908 . Physikalisches Staatslaboratorium.

\section{ELEKTRISCHER OFEN}

\section{Von Dipl.-Ing. H. Hanemann, Charlottenburg').}

Die durch den elektrischen Strom mit gekörnter Kohle als Widerstandsmasse geheizten

I) Mitteilung aus dem eisenhüttenmännischen Institut der Königl. Technischen Hochschule.
Oefen dürfen nur so weit erhitzt werden, als es die Feuerfestigkeit der Materialien zuläBt, aus denen der Heizraum gebildet ist. Sieht man von dieser praktischen Begrenzung ab, so ist die Möglichkeit der Temperatursteigerung bedingt 